\title{
Probing the nuclear equation of state in heavy-ion collisions at Fermi energy in isospin-sensitive exclusive experiments
}

\author{
P.Napolitani ${ }^{1,2}$, M.Colonna ${ }^{3}$,F.Gulminelli ${ }^{1}$, E.Galichet ${ }^{2,5}$, S.Piantelli ${ }^{4}$, G.Verde ${ }^{3}$, and E.Vient ${ }^{1}$ \\ 1 LPC Caen, ENSICAEN, Université de Caen, CNRS/IN2P3, 14050 Caen cedex 4, France \\ 2 IPN, CNRS/IN2P3, Université Paris-Sud 11, 91406 Orsay cedex, France \\ ${ }^{3}$ INFN-LNS, Laboratori Nazionali del Sud, 95123 Catania, Italy \\ 4 Sezione INFN di Firenze, Via G. Sansone 1, 50019 Sesto Fiorentino, Italy and \\ 5 Conservatoire National des Arts et Métiers, 75141 Paris Cedex 03, France
}

For the FAZIA collaboration

\begin{abstract}
We propose a few selected experimental approaches to show that new-generation instruments can give a direct access to significant observables on the density dependence of the symmetry energy in the nuclear equation of state. The form of such dependence is investigated within the Stochastic Mean Field model, coupled to a secondary-decay treatment.
\end{abstract}

\section{INTRODUCTION}

The exotic-beam facilities which already exist, or which are under construction, invite to focus on a longstanding experimental challenge: heavy-ion collisions induced by exotic nuclei at low and intermediate energies will be a probe for the properties of isospinasymmetric nuclear matter [1]. In particular, numerous next-generation experiments will be dedicated to the study of the density behaviour of the symmetry energy.

Such quantity defines the isospin-asymmetric part of the equation of state (EOS), which can be deduced from different phenomenological parametrisations [2 -4]. The corresponding predictions can be grouped schematically into two main forms: either a 'stiff' or a 'soft' dependence of the symmetry energy as a function of the nuclear density [4, 5]. At present, considerable effort is dedicated to pin down the density dependence in the regime of relativistic heavy-ion reactions [3, 4, 6], where supersaturation densities are accessible. Such conditions determine a significant difference between the two forms for the density dependence but the uncertainties in the hadron effective mass splittings and momentum dependence in the isovector channel introduce new degrees of freedom, enhancing the complexity of the problem [7].

These complications do not arise in the Fermi-energy regime, where the functional form of the symmetry energy at subsaturation densities should in principle be accessible. Furthermore, these conditions give access to the interesting issue of the influence of cluster correlations [8, 9]. At Fermi energies, the difficulty is that the difference between the theoretical predictions for the density dependence is less pronounced than at the large supersaturation densities which can be probed at relativistic energies: in particular, it is not always clear whether an effective discrimination between different degrees of asy-stiffness is really possible. On the one hand, this is due to the dependence of the predictions on the existing transport models [3, 7, 10]. On the other hand, besides the detection limitations, also the secondary decay contributes in deforming EOS-sensitive isotopic ob- servables 11 13]: we will discuss how this can affect the comparison protocol between model and experiment.

In this respect, we need to identify isotopic observables which, first of all, manifest a significant sensitivity to the change between the different asy-EOS forms and, in addition, constitute robust experimental observables against the effect of secondary decay and detection limitations [10, 14 16], is necessary. We also indicate that, recently, such study has profited from the results of isospin-transport experiments, which already imposed theoretical constraints [5, 17 21]. This work is aimed to give a schematic guideline for measuring a selection of isospin observables with new-generation isospin-sensitive instruments, in comparison with former isospin-blind devices. In particular, within our simulation protocol, we focus on a forthcoming $4 \pi$ detector, FAZIA [22], explicitly planned for measuring isospin observables and its isospin-blind ancestor, INDRA [23, 24]. We only focus on these two detectors because they are well suited for representing two opposite situations. However, it is evident that they can not resume the several experimental strategies which exist or are planned and which are based on different innovative tools for accessing the isospin observables. In particular, the $4 \pi$ detector CHIMERA [25] is a new-generation detector which is rapidly improving and evolving between these two extremes and its experimental results already mark the way towards future isospinsensitive devices.

\section{DESCRIPTION OF THE MODEL}

In this paper, a collision system is described within the Stochastic Mean Field (SMF) model. This is a time-dependent semi-classical mean-field model where nucleon-nucleon collisions as well as fluctuations are taken into account in the Boltzmann-Uehling-Uhlenbeck formalism (see [26] and references therein); the references [4, 27] give theoretical and numerical details. Such model is reliable in the Fermi-energy regime, for an incident energy ranging between about 10 and $200 \mathrm{MeV}$ 

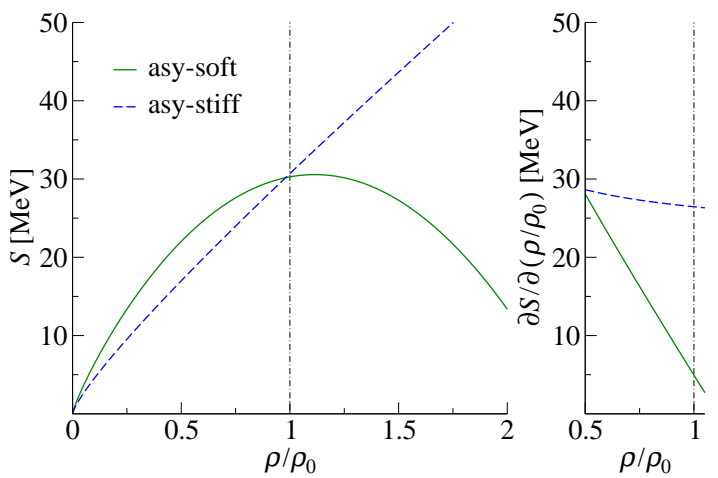

FIG. 1: (Color online) Left. Asy-soft and asy-stiff forms for the symmetry energy. Right. Corresponding derivative with respect to the density in the low-density region

per nucleon. In particular, due to the realistic isospindependent mean-field and the introduction of density fluctuations, the model gives a reliable description of heavy and intermediate-mass fragment properties; since elastic collision processes are accounted for, the model also describes the production of preequilibrium neutrons and protons reliably; nevertheless, the description of light particles demands correlations which are out of the scope of the model.

In the calculations, we adopt a parametrisation which gives the same properties as the $\mathrm{SkM}^{*}[28]$ force for nuclear matter: these are a saturation density $\rho_{0}=$ $0.16 \mathrm{fm}^{-3}$ and an incompressibility modulus $K_{\infty}=$ $200 \mathrm{MeV}$ which corresponds to a soft equation of state. We adopt two different prescriptions for the behaviour of the symmetry energy $S$, which are respectively:

$$
\begin{aligned}
S_{\mathrm{stiff}} & =a\left(\frac{\rho}{\rho_{0}}\right)^{2 / 3}+\frac{b}{2} \frac{\rho}{\rho_{0}}, \\
S_{\mathrm{soft}} & =a\left(\frac{\rho}{\rho_{0}}\right)^{2 / 3}+\frac{1}{2}\left(c \rho+d \rho^{2}\right),
\end{aligned}
$$

with the parameters $a=12.7 \mathrm{MeV}, b=36 \mathrm{MeV}, c=$ $481.7 \mathrm{MeV} \mathrm{fm}^{3}$, and $d=-1638.2 \mathrm{MeV} \mathrm{fm}{ }^{6}$. As illustrated in fig. [1] the first is an 'asy-stiff' form, for which the potential symmetry term linearly increases with nuclear density, while the second is an 'asy-soft' form, corresponding to a flatter behaviour of the potential symmetry energy around and below normal density. More details are provided in Ref.[4].

Isospin effects originate from the fact that neutrons and protons experience different forces. In particular, the difference between the neutron and proton currents $\boldsymbol{j}_{n}-\boldsymbol{j}_{p}$, that develop in presence of asymmetry $(\nabla I)$ and/or density $(\nabla \rho)$ gradients, is stricly connected to the strength of the symmetry energy (and of its derivative). In fact, within a simple hydrodynamical picture, one can write:

$$
\boldsymbol{j}_{n}-\boldsymbol{j}_{p} \propto \underbrace{S(\rho) \nabla I}_{\text {diffusion }}+\underbrace{\frac{\partial S(\rho)}{\partial \rho} I \nabla \rho}_{\text {migration }} .
$$

Hence, in presence of asymmetry gradients (diffusive processes) we test essentially the strength of the symmetry energy while, when density gradients are encountered along the dynamical path, we observe "isospin migration" towards the low density regions, ruled by the derivative of the symmetry energy.

Within the SMF model, the systems ${ }^{68} \mathrm{Ni}+{ }^{68} \mathrm{Ni}$, ${ }^{58} \mathrm{Ni}+{ }^{68} \mathrm{Ni}$ and ${ }^{58} \mathrm{Ni}+{ }^{58} \mathrm{Ni}$ at 15 and $40 \mathrm{MeV}$ per nucleon were simulated in the present work for a continuous distribution of impact parameters $b$, which evolves as $b \mathrm{~d} b$.

Fig. 2 shows the reaction mechanisms observed as a function of the reduced impact parameter $b_{\text {red }}$ (normalised to the sum of the nuclear radii of the target and projectile nuclei). Fusion reactions (F) were identified from the presence at the asymptotic time of a unique fragment having bigger mass than the projectile (or the target, equivalently); the quasi-elastic channel is defined as non-fusion events where the sum of the charges of the two largest fragments exceeds a large fraction of the charge of the projectile (or the target, equivalently; this fraction is adjusted to $90 \%$ and $85 \%$, for the incident energies of 15 and $40 \mathrm{AMeV}$, respectively); the neck contribution (neck) is recognised from events which do not belong to the previous categories and where at least three fragments have larger charge than helium; all the other events are interpreted as dissipative binary reactions (DI). This figure shows that a centrality selection corresponds only approximately to a selection of the reaction mechanism. This is especially true for the neck events, which never dominate the total cross section. Since the experimental apparatus does not deliver the same response to the different reaction mechanisms, this may result in an uncontrolled bias if unfiltered simulations are confronted to the experimental data.

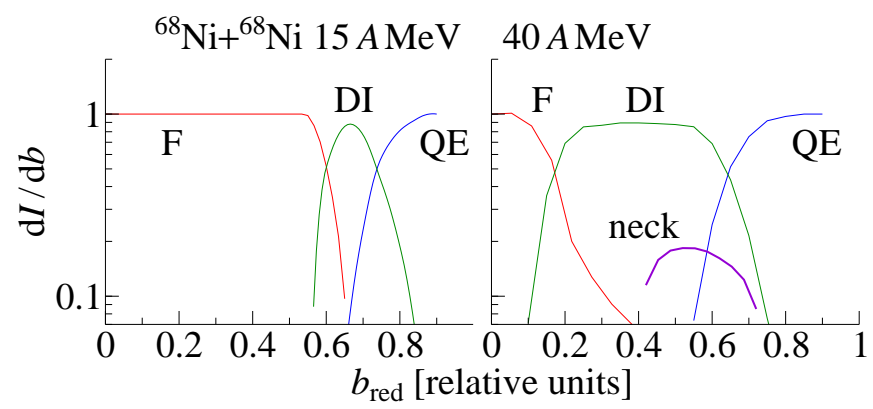

FIG. 2: (Color online) Probability for different reaction mechanisms as a function of the reduced impact parameter for the systems ${ }^{68} \mathrm{Ni}+{ }^{68} \mathrm{Ni}$ at 15 (left panel) and 40 (right panel) $A \mathrm{MeV}$. The asy-soft (here used) and asy-stiff (not shown) forms give very close results. 


\section{A. Evolution of the isospin content and effect of the secondary decay}

In the calculations, the dynamical stage is followed till the time $t=260 \mathrm{fm} / \mathrm{c}$. In order to deal with the full range of impact parameters which are simulated, the fragment properties are evaluated as soon as the system breaks up into pieces within this interval of time, in each event. This procedure implies that events with only one fragment (incomplete fusion) are followed until the final (longer) time, leading to a reduction of the calculated size and excitation energy of the compound system, due to nucleon emission. The output of the SMF calculation is then coupled to the evaporation model GEMINI 29] (the 2003 release is used). This coupling is necessary because of two reasons. First, secondary decay extends over a much longer time than the numerical stable interval of time of any transport code; Second, even moleculardynamics models, which in principle can describe the production of complex light particles, can not reproduce the statistical branching ratios of compound-nucleus emission [30].

We shall emphasise that in the absence of secondary decay, a basic quantity like the isotopic composition of intermediate-mass fragments (IMF) would be sufficient to distinguish between the asy-stiff and asy-soft behaviour of the symmetry energy, even in stable systems. This is shown in fig. 3 (upper spectra labelled 'SMF'), where the average neutron number to atomic number ratio of the biggest fragment in all events not issued of fusion is plotted as a function of the corresponding longitudinal component of the rapidity in the centre of mass, relative to the projectile (target) rapidity for positive (negative) rapidities, for the two systems ${ }^{58} \mathrm{Ni}+{ }^{58} \mathrm{Ni}$, ${ }^{58} \mathrm{Ni}+{ }^{68} \mathrm{Ni}$ and ${ }^{68} \mathrm{Ni}+{ }^{68} \mathrm{Ni}$ at 15 and $40 \mathrm{AMeV}$. In the symmetric systems, the fragments are more neutron rich in the asy-stiff case and the effect enhances for the more neutron-rich system. This scenario can be attributed to the lower value of the symmetry energy below normal density in the asy-stiff case, with respect to the asy-soft form (see fig. 1). In the asymmetric system, in addition to nucleon emission, isospin diffusion takes place through the low density interface between the two reaction partners. As illustrated by eq 3 , diffusion is a process of isospin equilibration between the two asymmetric reaction partners which acts more effectively for higher values of the symmetry energy; at low densities, this process is therefore more effective for a softer form of the EOS, which is in this case given by the asy-soft parametrisation (see fig. 11). As a consequence, the projectile and target sides in the diagram of fig. 2 are more similar in this case, and the difference between the two parametrisations is particularly relevant at midrapidity, for the system at $40 \mathrm{AMeV}$

If the reaction ended at this stage in the laboratory, there would be no need to introduce complex differential observables [17], or to exploit exotic radioactive beams for the purpose of this investigation.
Nevertheless, unfortunately, the secondary decay makes the discrimination between the two parametrisations difficult: in stable and moderately exotic systems the secondary decay can wash out most of the discriminating signals; this effect is illustrated in fig. 3 (lower spectra labelled 'SMF+GEMINI'), where the hot system described within the SMF model is made decay by the GEMINI model.

We can not exclude that such a prominent effect may depend on the model or the system chosen; different contributions of secondary decay have been reported in the literature [11 14, 18] and no systematic study has been performed to our knowledge. However, this prologue should drive the attention on the fact that the direct measurement of observables related to the equation of state is certainly challenging. In particular, the evaporation process has the effect of modifying and even smearing out

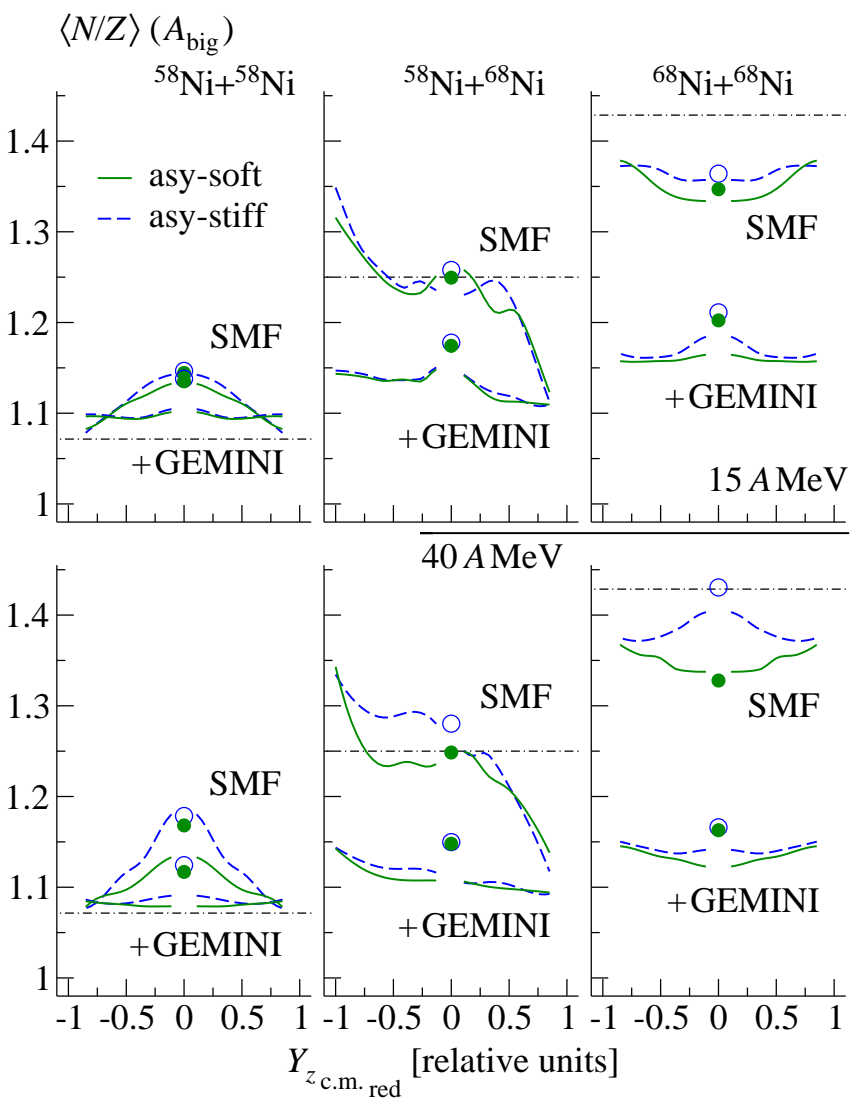

FIG. 3: (Color online) Variation of the isotopic composition of the biggest fragment (excluding fusion events) as a function of the rapidity in the centre of mass relative to the projectile (target) rapidity for positive (negative) rapidities calculated with both the asy-stiff and asy-soft parametrisations before (SMF, upper spectra) and after (SMF+GEMINI, lower spectra) secondary decay for the systems ${ }^{58} \mathrm{Ni}+{ }^{58} \mathrm{Ni}{ }^{58} \mathrm{Ni}+{ }^{68} \mathrm{Ni}$ and ${ }^{68} \mathrm{Ni}+{ }^{68} \mathrm{Ni}$ at $15 \mathrm{AMeV}$ (upper panels) and $40 \mathrm{AMeV}$ (lower panels), for all impact parameters. The dots indicate fusion nuclei. The dashed lines indicate the average isotopic composition of the colliding system. 
the observables we wish to investigate. Beside achieving an increasingly more precise understanding of the decay sequence (especially in exotic systems), we should also define efficient strategies in choosing the nuclear system, adopt new observables and refine the criteria of event selection to reduce the effect of the secondary decay, so as to preserve significant signatures. These requirements also impose to develop innovative experimental strategies.

\section{B. Detection}

In the present report we suggest some possible solutions which would require the use of a dedicated detector device. Such detector, which is under development within the FAZIA project ('Four- $\pi A$ and $Z$ Identification Array' [22]), is a $4 \pi$-array of telescopes, each one designed for measuring the kinetic energy, the nuclear charge and the mass of the intercepted fragments (the mass measurement over an extended mass range constitutes the main innovation for such device). An additional calculation filters the results produced by the reaction model $(\mathrm{SMF}+\mathrm{GEMINI})$ by accurately simulating the functioning of the FAZIA detector, including the detailed geometry and the response of the different detection modules [31]. In particular, in the case of FAZIA, the telescope is composed by two silicon detectors of $300 \mu \mathrm{m}$ and $500 \mu \mathrm{m}$ respectively and a CsI detector; if a particle is arrested in the second silicon detector, the kinetic energy and the time of flight are measured. In this case, the particle can be identified in nuclear charge and, if possible, in mass by the correlation of the energy versus the energy loss; in the simulation the measured nuclear charge is then set equal to the theoretical value, and the mass is set equal to the theoretical value up to phosphor and, for higher elements, it is deduced from the kinetic energy and the time of flight.

The functioning of the former-generation detector $I N$ $D R A$ [23, 24] is also simulated for comparison. FAZIA differs from INDRA because the granularity is larger (with a substantial gain in angular resolution) and because the mass, in addition to the nuclear charge, can be measured. When necessary, we will also simulate in the calculation the existence of neutron detectors imagined as a belt of $D E M O N$ [32] telescopes covering all azimuthal angles and disposed on the longitudinal plane (containing the beam axis). For such a configuration, we do not take into account the effect of the charged-particle detector on the neutron detection in the $D E M O N$ modules.

In this report, we compare two exclusive experimental approaches for reaction experiments with exotic nuclei. The first employs INDRA (with, if needed, the addition of $D E M O N$ modules), where the mass of the fragments (and any isotopic observable) is not measured, but deduced from the nuclear charge through the use of a parametrization (EPAX, which supposes that the cold nuclei are attracted towards the residue corridor), which is closely compatible with the decay model GEMINI and which was adjusted to mostly stable (or moderately exotic) nuclei. The second approach is FAZIA (with, eventually, the addition of $D E M O N$ modules), which delivers a direct measurement of the masses, independently of the exoticity of the system. Hence the comparison with the response of INDRA is useful to appreciate up to which extent, for the experiment and the observables under examination, fragment masses may deviate from the residue corridor and, once measured by FAZIA, bring information on the isovector term of the nuclear interaction.

\section{Statistics}

The number of events considered in the transport calculations are 1000. Each one of these events is then used to produce ten decay paths. For the purpose of simulating the experiment, since the geometrical efficiency in the selection of complete events could impose a severe reduction of the statistics, the number of events is then largely increased by considering rotations around the beam axis. At the end, the simulated values, within the uncertainties, correspond to one standard deviation of the mean, calculated for a statistics of 10000 events (1000 transport events multiplied by 10 decay paths) and considering the full error propagation. We would like to stress that the simulated results we give should be considered as a lowerlimit expectation with respect to a real experiment. The reason is that in a real experiment we can profit from advanced tools of event selection and develop the data analysis down to fine details, while in the simulation the reduced statistics allows to simulate the data analysis only up to a limited degree of accuracy. This limitation also propagates to the error bars of the simulated results.

For this same reason, we did not exploit the full set of simulations. In fact, in some cases (especially for the neutron-poor systems at the lower beam energy) the uncertainties were too large, as compared to the different predictions of the two asy-EOS. We stress again that this would not correspond well to the experimental situation, which is already successfully progressing on the study of isospin effects with stable beams. Moreover, for the purpose of comparison with experimental data, the accuracy of the simulated results can be improved by increasing the number of SMF events considered.

\section{SIMULATIONS: FROM THE SELECTION OF THE IMPACT PARAMETER TO THE ISOTOPIC OBSERVABLES}

Experimentally, the impact parameter can be deduced from the transverse energy per nucleon $E_{\mathrm{tr}}$ of light particles with $Z<3$ (it should be precised that in the SMF simulations no particles with $Z=2$ are produced; they are generated in the decay, by GEMINI). The correlation 
between the impact parameter and the transverse energy

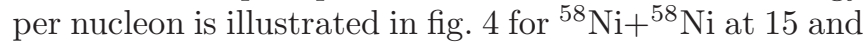
$40 \mathrm{AMeV}$. At $40 \mathrm{AMeV}$, the correlation extends over all impact parameters except for $b_{\text {red }}<0.2$, where fusion becomes the dominant mechanism (see fig. 22). At $15 \mathrm{AMeV}$, the correlation is still valid for peripheral collisions, but the sensitivity is gradually lost when entering the fusion regime $\left(b_{\text {red }}<0.55\right)$ and the dissipation becomes total for complete fusion (around $b_{\text {red }}<0.3$ ). In fig. 4 the measurement of such correlation is simulated for the two detector arrays INDRA and FAZIA: the measured transverse energy per nucleon is divided by the geometric efficiency $e_{\text {geo }}\left(I N D R A: e_{\text {geo }}=0.88, F A Z I A: e_{\text {geo }}=0.78\right)$. Both INDRA and FAZIA perform equivalently well. We shall emphasise that this implies that this same conclusion extends to any assembly where a part of one array is replaced by the corresponding part of the other, without reducing the whole angular coverage.

Profiting of this selection, we focus on the study of the isotopic composition of the largest fragments. This study, presented in fig. 5, is one example intended to answer the question whether the advantage of disposing of exotic systems could counterbalance the effect of the evaporation process. In fig. 吕, three systems ${ }^{58} \mathrm{Ni}+{ }^{58} \mathrm{Ni}$, ${ }^{58} \mathrm{Ni}+{ }^{68} \mathrm{Ni}$ and ${ }^{68} \mathrm{Ni}+{ }^{68} \mathrm{Ni}$ at two incident energies are studied; to keep an approximate link with the underlying reaction mechanism (see fig. 21), central events are defined as $b_{\text {red }}<0.2\left(b_{\text {red }}<0.55\right)$ for the incident en-

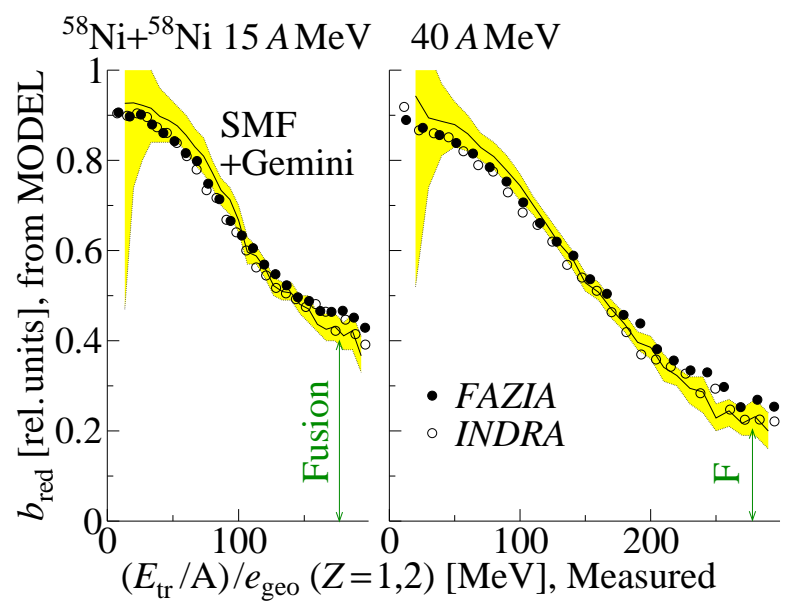

FIG. 4: (Color online) Correlation between the impact parameter and the transverse energy per nucleon for ${ }^{58} \mathrm{Ni}+{ }^{58} \mathrm{Ni}$ at 15 and $40 \mathrm{AMeV}$, as simulated by SMF+GEMINI (solid lines). For comparison, the same correlation is simulated for the two experimental approaches INDRA and FAZIA, where the transverse energy per nucleon is further divided by the geometric efficiency $e_{\text {geo }}$. The interval in impact parameter which corresponds to fusion is indicated. The statistical uncertainties of the calculation SMF+GEMINI (asy-soft form) are indicated by the coloured bands; they are reflected in the filtered data with comparable magnitude (Not indicated in the figure, for better visibility)

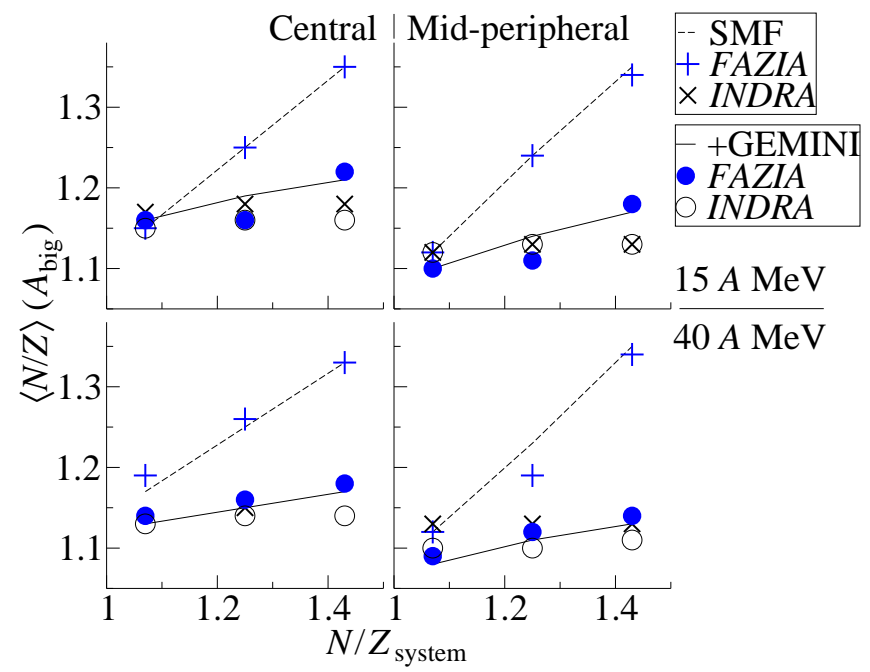

FIG. 5: (Color online) Isotopic composition of the largest fragments as a function of the isotopic composition of the system at 15 (upper panels) and $40 \mathrm{AMeV}$ (lower panels), under the constraint of selecting either central (left panels) or mid-peripheral events (right panels). This observable is simulated for the hot system (SMF - asy soft) and for the cold system (SMF+GEMINI). For comparison, the same correlation is simulated for the two experimental approaches INDRA and FAZIA.

ergy of 40 (15) $A \mathrm{MeV}$; mid-peripheral events are defined as $0.25<b_{\text {red }}<0.6\left(0.6<b_{\text {red }}<0.75\right)$ for the incident energy of 40 (15) $A \mathrm{MeV}$. The process of evaporation (GEMINI) which affects the hot systems (SMF) is more relevant at higher incident energy. Thus, larger variations of the isotopic composition $(N / Z)$ of the largest residue $\left(A_{\mathrm{b} i g}\right)$ as a function of the isotopic composition of the system manifest at $15 \mathrm{AMeV}$. Experimentally, FAZIA would measure such trend correctly. Concerning INDRA, the redundancy between the parametrisation used to deduce the mass number from the atomic number (residue corridor), and the description of the decay by GEMINI globally results in an apparently good performance for stable systems; for such systems the evaporation process ends in populating the residue corridor. However, such effect depends on the model. In particular, for the most neutron-rich system, the mass number parametrisation reveals to be no more sufficient and a direct measurement of the masses is necessary [33, 34]. Such parametrisation will be even less adequate for exotic systems (which will be studied in future installations exploiting exotic beams), when the residue corridor is not reached. To give an extreme example, we applied the experimental filters also to the hot systems (SMF), neglecting the decay. FAZIA measures correctly even these very neutron rich systems, while $I N D R A$ associates the residue-corridor masses to the atomic numbers and gives almost the same result which was previously simulated for the cold systems (SMF+GEMINI).

In conclusion, in most studies of isospin effects on the 
equation of state, or of thermodynamical properties in the decay, exotic systems are highly desirable; otherwise, the smearing effect of the evaporation process will be difficult to surmount.

\section{THE EQUATION OF STATE: THREE EXPERIMENTAL APPROACHES}

The observables studied in fig. 5 led to the conclusion that secondary decay may impose major difficulties in the experimental study of the equation of state. Exotic nuclei may be advocated as more suited systems, where the secondary decay may be better retraced as far as the residue corridor is not reached in the cooling process. Alternatively, we can also search for other observables which are less sensitive to the secondary decay. Three possibilities are explored in this section: the imbalance ratio, first introduced by the MSU group [17, 19]; the high-energy part of neutron and proton spectra, proposed in the framework of BUU 35], and also successfully exploited via IQMD [36]; and finally, the neck emission in ternary events, studied at length by the Catania group [4, 37].

\section{A. Imbalance ratios}

The isospin-transport ratio, introduced by F.Rami 38] to study isospin equilibration, has been exploited by the MSU group [10, 17, 19] because of its sensitivity to the density dependence of the symmetry energy. In the case of the $\mathrm{Ni}$ isotopes of the present study, the imbalance ratio is defined as

$$
R=\frac{2 x_{58+68}-x_{68+68}-x_{58+58}}{x_{68+68}-x_{58+58}},
$$

where $x$ is an isospin sensitive observable, possibly linearly correlated with asymmetry, measured in the three different reactions. The motivation of introducing such observable is that, by focusing on the differences in isospin observables between mixed and symmetric systems, $R$ is expected to largely remove the sensitivity to preequilibrium emission and enhance the sensitivity to isospin diffusion between projectile and target. We have undertaken this analysis for the reactions at $40 \mathrm{AMeV}$, fow which the statistics of binary events is good enough.

Considering all impact parameters, we assigned the average isospin content of the largest fragment, not produced in fusion events, to the $x$ variable. This observable, explored in fig 3, is shown again in fig 6 together with the corresponding response of the experimental device FAZIA. From a closer inspection of figs 36, one may notice that the effect of the secondary decay on this observable is not the same in the different rapidity bins. This can be ascribed to the fact that the excitation energy of primary fragments is not constant, but it is larger at mid-rapidities, which correspond to more central events.
However, in each rapidity bin, this observable is expected to be correlated to its value prior to decay. From these data points fig 7 is constructed. We may remark that, since the isotopic composition of the asymmetric system is intermediate between the symmetric systems, according to the definition of $\mathrm{Eq} 4$, the imbalance ratio should approach zero in the vicinity of midrapidity and evolve towards negative values in the projectile side for increasing rapidity and eventually reach the value of -1 for large transparency; in the target side, the imbalance ratio should evolve for increasing negative rapidity towards the same values explored in the projectile side with opposite sign. This overall behaviour, reflected by the hot fragments (SMF), is well reproduced also after the evaporation process (SMF+GEMINI); this shows that, indeed, the effects of secondary decay can be removed when suitable observables which combine differences and ratios of isospin dependent properties are adopted, such as the imbalance ratio. The difference between the predictions of the asy-stiff and asy-soft parametrisations is preserved after the secondary decay stage and is measurable with FAZIA. Comparing fig. 6 and fig. 7 we can see that the discrimination between the two parametrisations is essentially due to the presence of the neutron rich system ${ }^{68} \mathrm{Ni}+{ }^{68} \mathrm{Ni}$, which is the only case where the residue corridor is not reached during the deexcitation. Following the discussion of fig. 5 , we expect that the imbalance ra-

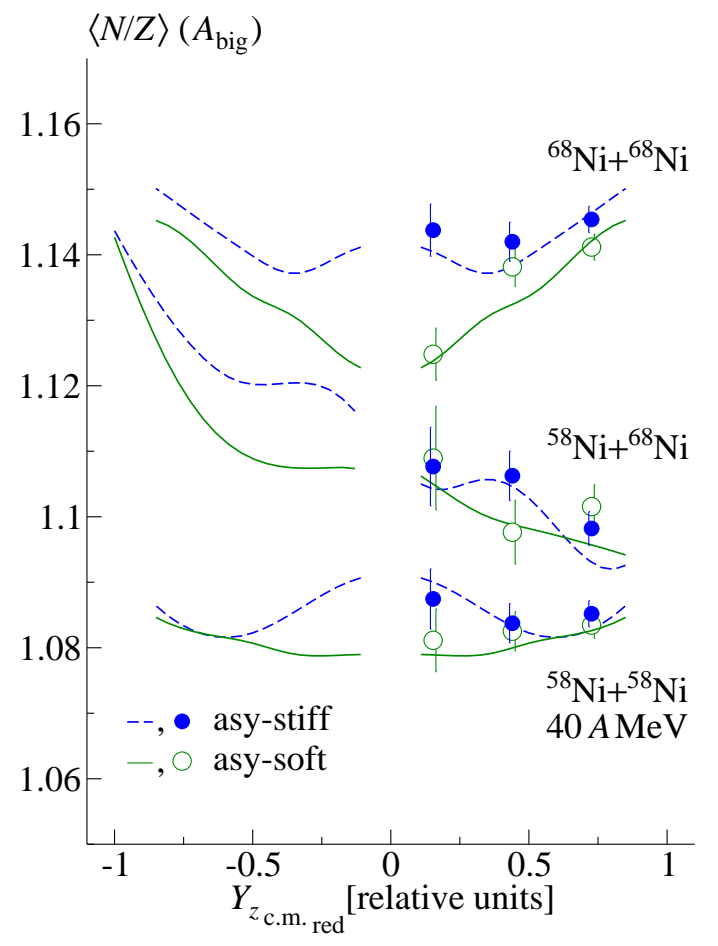

FIG. 6: (Color online) Same observable studied in fig 3, we focus on the cold systems (SMF+GEMINI) of the system at $40 A \mathrm{MeV}$ and we add the filtered data, simulated for the experimental device $F A Z I A$. 


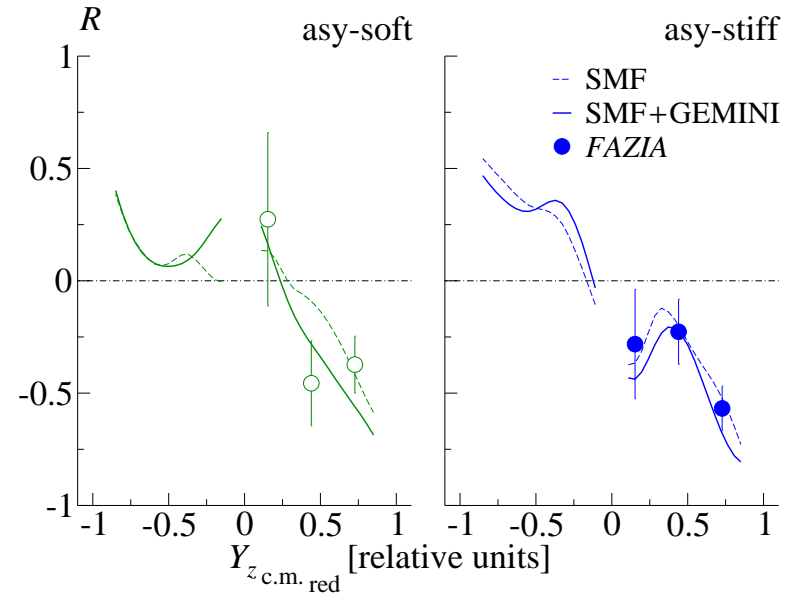

FIG. 7: (Color online) Imbalance ratio (see text) for the asy-stiff and asy-soft parametrisations, corresponding to the same observable studied in fig 3, for the systems ${ }^{58} \mathrm{Ni}+{ }^{58} \mathrm{Ni}$ ${ }^{58} \mathrm{Ni}+{ }^{68} \mathrm{Ni}$ and ${ }^{68} \mathrm{Ni}+{ }^{68} \mathrm{Ni}$ at $40 \mathrm{AMeV}$, for all impact parameters, and as a function of the longitudinal component of the rapidity in the centre of mass relative to the projectile (target) rapidity for positive (negative) rapidities. The statistical uncertainties of the model are indicated by the error bars.

tio will provide a better discrimination between different effective interactions with the use of more exotic beams.

A similar analysis could be performed taking the transverse energy $E_{\mathrm{tr}}$ of light particles as a centrality selector (see fig. 4) instead of the rapidity of the largest fragments. However, the latter appears more directly connected to the dissipation mechanism between the two reaction partners, while $E_{\mathrm{tr}}$ may be influenced by additional effects, such as pre-equilibrium dynamics and cluster-emission mechanisms. For this reason, the analysis proposed in fig. 7 should be more helpful when comparing experimental data with the predictions of transport models which differ by the treatment of correlations [27]. Also different isospin-sensitive observables may be used, such as the isoscaling parameter and the isobaric yield ratio $\ln \left(Y\left({ }^{7} \mathrm{Li}\right) / Y\left({ }^{7} \mathrm{Be}\right)\right)$ employed by the MSU group [10], or the average $N / Z$ of the light-chargeparticle emission [18]. This would be possible within our analysis protocol, but it demands a larger statistics for the secondary-decay treatment. For all those choices, as far as the observables are linearly correlated to the $\mathrm{N} / \mathrm{Z}$ of projectile-like and target-like fragments [10, 18], the corresponding results for the imbalance ratio should be rather close to the ones depicted in fig. 7 for the observable we adopted.

\section{B. Light particles: neutron and proton spectra}

The evolution of isotopic ratios of light particles as a function of their kinetic energy and the isotopic composition of the system $(N / Z)_{\text {system }}$ has also been shown to be sensitive to the stiffness of the equation of state [35, 36]. When light particles (like $t$ and ${ }^{3} \mathrm{He}$ ) are concerned, the SMF model can no more access such observable which, in general, is strongly dependent on the model. For these reasons, in this section we restrict to the study of preequilibrium neutrons and protons.

The neutron and proton yields as a function of the perpendicular component of the kinetic energy $E_{\perp}$, are illustrated in fig. 8 for the system ${ }^{58} \mathrm{Ni}+{ }^{68} \mathrm{Ni}$ at 40 $A M e V$. The spectra are simulated before (SMF) and after (SMF+GEMINI) the secondary decay. As expected, the low-energy side of the spectra is mostly affected by the secondary decay. To minimise the effect of secondary decay, we select the high-energy side of the spectra where the two simulations, before and after evaporation, coincide: we impose $E_{\perp}>25 \mathrm{MeV}$.

The shape of the proton spectra can be measured directly by an experimental device like FAZIA, as simulated in fig. 8, or like INDRA. The shape of the neutron spectra can be deduced from the proton spectra only if complete events can be recorded; since this is practically impossible to achieve at intermediate energies due to the high multiplicity of the events, the neutron spectrum should be measured directly with the use of a neutron detector; in the present simulation, shown in fig. 8, we suppose that the detector $D E M O N$ is employed. In particular, we suppose to dispose of $D E M O N$ telescopes covering all azimuthal angles, and only an incomplete interval of $30 \mathrm{deg}$ in zenith angle, so that the shape of neutron spectra can be measured correctly. For such a configuration, we do not take into account the effect of the charged-particle detector on the neutron detection, even if, in a realistic configuration, such effect

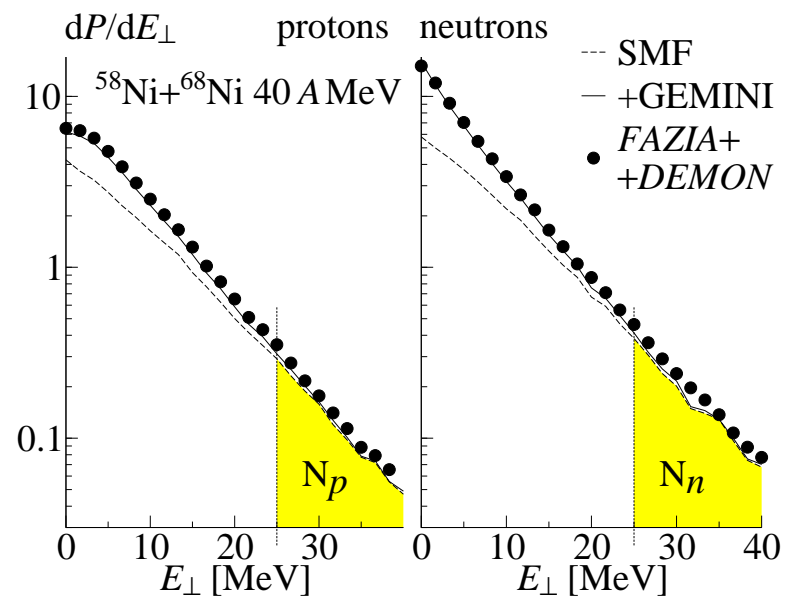

FIG. 8: (Color online) Proton (left panel) and neutron (right panel) yields as a function of $E_{\perp}$ for the system ${ }^{58} \mathrm{Ni}+{ }^{68} \mathrm{Ni}$ at $40 \mathrm{AMeV}$. Units are in percent of the total neutron yields. The spectra are simulated for the hot system (SMF - asy-soft) and for the cold system (SMF+GEMINI). The same spectra are simulated as measured with an experimental device composed of FAZIA coupled with DEMON modules. 
could be large and the neutron detectors may have to be even substituted to some charged-particle detectors. It is possible, but it should be proved experimentally, that analogous information may be deduced from the analysis of $t{ }^{3} \mathrm{He}$ spectra, which would make this analysis accessible to neutron-blind devices like INDRA or FAZIA alone. The integral of the proton spectra can be measured directly, by selecting only complete events. The integral of the neutron spectra can not be measured directly in any case: in general, the efficiency of neutron detectors would not be sufficient to impose a condition of event completeness even when using a $4 \pi$-array. The integral of neutron spectra is deduced by difference, from the measurement of the nuclear charge and mass of fragments and particles detected in complete events. Hence, for a correct evaluation of the efficiency of the neutron detector, also the mass detection, as provided by FAZIA, is necessary. The slight discrepancy between the theoretical and measured spectra in the simulation of fig. 8, both for neutron and proton spectra, is due to the choice of defining as complete events those events where less than $15 \%$ of the total nuclear charge is missing.

From the neutron and proton spectra we deduce the evolution of the ratio between the number of neutrons over the number of protons $\mathrm{N}_{n} / \mathrm{N}_{p}$, averaged over complete events and normalised to the isotopic composition

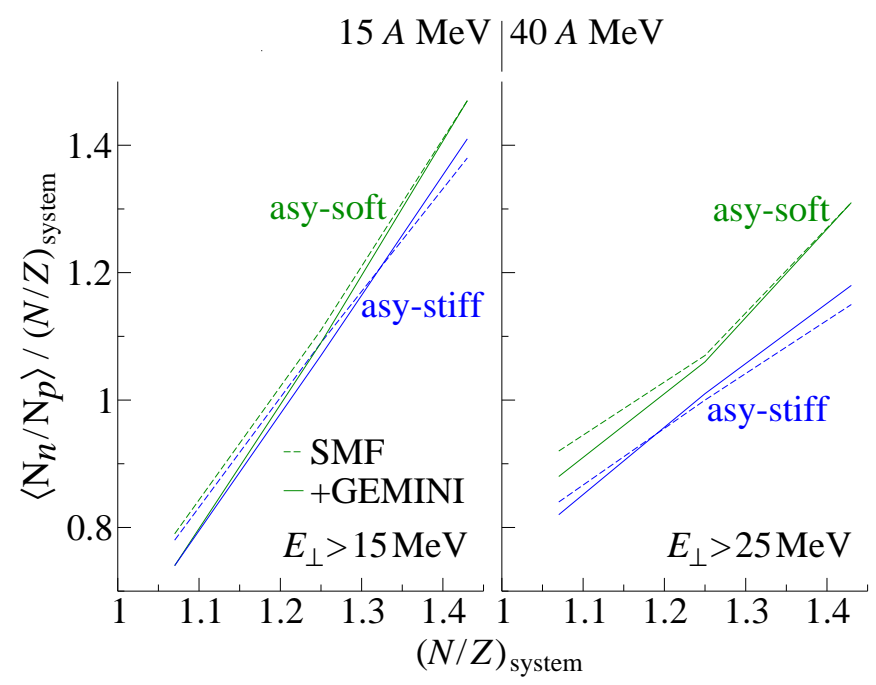

FIG. 9: (Color online) Ratio between the number of neutrons over the number of protons averaged over complete events and normalised to the isotopic composition of the system, as a function of the isotopic composition of the system. This observable is simulated for incident energies of 15 and $40 \mathrm{AMeV}$, for hot systems, before the secondary decay occurs (SMF) and for cold systems, after the secondary decay (SMF+GEMINI). The two prescriptions for the equation of state, asy-stiff and asy-soft are employed. Specific event selections are imposed depending on the incident energy. $15 \mathrm{AMeV}$ : only neutrons and protons with $E_{\perp}>15 \mathrm{MeV}$ are chosen. $40 \mathrm{AMeV}$ : only neutrons and protons with $E_{\perp}>25 \mathrm{MeV}$ are chosen. In both cases, only events not leading to fusion are selected. of the system $(N / Z)_{\text {system }}$, as a function of the isotopic composition of the system. In fig. 9 this observable is studied for hot systems, before and after the secondary decay, for the two incident energies 15 and $40 \mathrm{MeV}$ per nucleon. We mention that the condition $E_{\perp}>15 \mathrm{MeV}$ is used for the reactions at $15 \mathrm{AMeV}$, due to the lower energy available in this case for the pre-equilibrium emission. It has been already observed that the best sensitivity to the equation of state is obtained in central collisions 35. However, especially at $15 \mathrm{MeV}$ per nucleon, it is difficult to extract central events from the $E_{\text {tr }}$ distribution, see fig. 4. Hence, for both incident energies, we consider all impact parameters, but we select fusion events out. The different parametrisations for the equation of state, asy-stiff and asy-soft, do not determine any appreciable difference at $15 \mathrm{MeV}$ per nucleon. This could be due to the fact that, at this low energy, compression-expansion effects are quite reduced and, while pre-equiquilibrium emission takes place, the nuclear density keeps rather close to the saturation value, where the two parametrisations give, by construction, the same value of the symmetry energy. On the other hand, at $40 \mathrm{MeV}$ per nucleon, the two parametrisations of the symmetry energy lead to clearly distinct behaviours even for non-exotic systems: the normalised average neutronto-proton ratio is up to $13 \%$ larger for the asy-soft case. Now the isotopic content of the pre-equilibrium emission is sensitive to the low-density behaviour of the symmetry energy, since particles mostly escape while the composite nuclear system is expanding. A neutron-richer emission is seen in the asy-soft case, corresponding to the higher value of the symmetry energy below normal density (see fig. (1). One can also notice that the dependence of this observable on the system initial asymmetry is flatter for the reactions at $40 \mathrm{AMeV}$. This reflects the higher energy available, with respect to the $15 \mathrm{AMeV}$ case, at the pre-equilibrium stage. In the following, we concentrate on the systems at $40 \mathrm{MeV}$ per nucleon. We observe that the signals are still preserved after the secondary decay, thanks to the energy selection we adopted. In fig. 10, we simulated the response of the two detection devices, FAZIA and INDRA. The error bars account for the accuracy in the correction for the efficiency of the neutron detection, supposing that it is known with an uncertainty of around 5\%; additional systematical uncertainties (not shown) would be produced by the procedure of accounting for the number of fragments and particles which are not detected.

The simulation demonstrates that FAZIA would perform efficiently and succeed in measuring the signal. However, after the deexcitation stage, the mass of the evaporation residues, which should be measured in order to reconstruct the number of emitted neutrons, approaches the residue corridor. In order to study a more constraining situation, for testing purposes, we replace the cold system by the hot system, before the decay, in the experimental filter. For the most neutron-rich system, we add simulated points representing INDRA, 


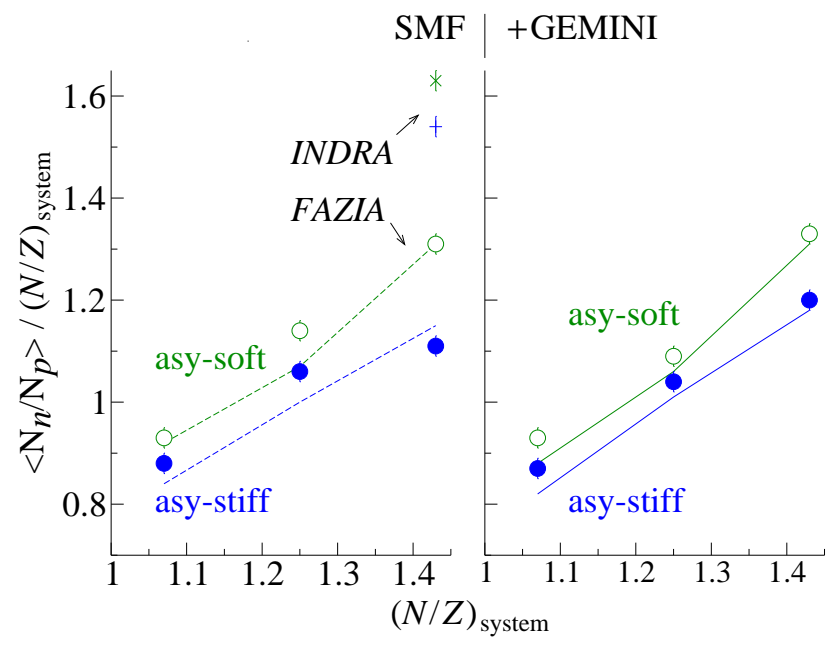

FIG. 10: (Color online) Same observable as in fig. 9 for an incident energy of $40 \mathrm{AMeV}$. Both hot systems (SMF, left panel) and cold systems (SMF+GEMINI, right panel) are simulated as measured with an experimental device composed of FAZIA coupled with DEMON modules before and after secondary decay. The simulation of INDRA coupled with $D E M O N$ modules is added for the most neutron-rich system, before secondary decay.

which does not measure the masses, but deduces them through the evaporation-corridor prescription introduced in the detector simulation: these points, as they are referred to a very neutron-rich system, far from the residue corridor, result in fact to be incompatible. We conclude that such signal survives after the secondary decay and is measurable only with a device like FAZIA in conditions where the reaction products are far from the residue corridor.

\section{Neck emission in ternary events}

It has been discussed in the literature [4] that the dissipative dynamics leading to neck formation in midperipheral collisions at Fermi energies is strongly sensitive to the isospin transport properties and to the isovector properties of the equation of state.

We focus on the system ${ }^{68} \mathrm{Ni}+{ }^{68} \mathrm{Ni}$ at $40 \mathrm{AMeV}$; such incident energy is large enough to exceed the threshold for the formation of neck fragments. For this specific simulation the stopping time was fixed to $160 \mathrm{fm} / \mathrm{c}$, which is long enough to follow the dynamics of the neck formation. We select ternary events where, in addition to light particles, we observe only the following three fragments: one quasi-projectile residue, one quasi-target residue, and one intermediate-mass fragment at midrapidity, which corresponds to the neck fragment. In addition, we impose two more constraints for the selection of ternary events. First, we reduce to semiperipheral collisions where $b_{\text {red }}$ varies in the interval $0.45<b_{\text {red }}<0.75$ : in fig. 2 we already signalled the presence of neck events in this interval. The second constraint is on the mass number of the third fragment: by adjusting a selection threshold to $Z \geq 5$, we ensure that the ternary events with three IMF's that we select can not be confused with secondarydecay events which produce fragments of small size.

The observable we investigate is the isotopic composition of the neck fragment in ternary events, selected as defined above. This system, studied before the secondary decay, is less neutron rich when the symmetry energy is parametrised with the asy-soft term, and more neutron rich when an asy-stiff interaction is used. This is due to isospin migration effects from projectile and target towards the low-density region of the neck. In particular, the evolution of the isospin asymmetry of the neck region is conditioned by the contribution of the migration process to the currents of neutrons and protons, driven by density gradients. Such contribution is represented in eq. 3 by the term proportional to the derivative of the symmetry energy and the density gradient. The process of migration determines an enrichment in the neutron content of the diluted neck region, which is larger for larger variations of the symmetry energy as a function of the density, and is therefore more effective for a stiffer form of the EOS around normal density, which is in this case given by the asy-stiff parametrisation (see fig. 1). When the secondary-decay process is added up, in the asy-soft case, the more excited neck fragments end up in the evaporation corridor and their $N / Z$ ratio reduces. In the asy-stiff case, the hot system is more neutron-rich and the decay path does not reach the evaporation corridor.

Such difference in shown in fig. 11] where the isotopic composition of neck fragments in ternary events is plotted as a function of $\phi_{\mathrm{AM}}$, where $\phi_{\mathrm{AM}}$ is the smallest angle which measures the angular misalignment between the velocity vector of the neck fragment in the centre of mass frame and the direction along which the two heaviest fragments (representing approximately the quasi-projectile and the quasi-target) are aligned. This convention, followed in this paper, does not correspond exactly to the definition of $\phi_{\text {plane }}$ [20], more often followed in experimental studies of the phenomenon of nonequilibrium fission. We signal that such studies are closely compatible with the present work and explicitly oriented to the investigation of the EOS.

The increasing trend observed in the asy-stiff case may be due to the impact parameter mixing in our analysis. Indeed, fragments with larger transverse velocities are more easily emitted in the most central events of the selected interval of impact parameters, for which, due to the longer reaction time, isospin migration is more effective. The trend is less clear in the asy-soft case, where isospin migration effects are less pronounced. For the same reason, fragments having $\phi_{\mathrm{AM}}$ close to $90 \mathrm{deg}$ are more excited. In the asy-stiff case, the isotopic composition of neck fragments reduces almost systematically when passing from the system before decay (SMF) and after decay (SMF+GEMINI). The effects related to the 
different excitation energy are more evident in the asysoft case: the less excited fragments experience a short decay path and are close to the isotopic composition of the hot fragments, while the fragments which could spend more excitation energy in the decay reached the evaporation corridor. The further addition of the experimental filter demonstrates that FAZIA would measure this observable precisely. On the other hand, INDRA would not succeed in measuring this observable, and the discrepancy is evident when the residue corridor is not reached, that corresponds to the asy-stiff case. In conclusion, the asymmetry of the neck fragments appears as a promising observable to be exploited in the investigation of the behaviour of the symmetry energy below saturation density. Due to the isospin-migration mechanism, these fragments are particularly neutron-rich, especially in the asystiff case, and the effect survives to the secondary-decay stage.

Isospin migration is more effective in neutron-rich systems, however interesting results have been reported also in the case of stable, less asymmetric systems, in theo-

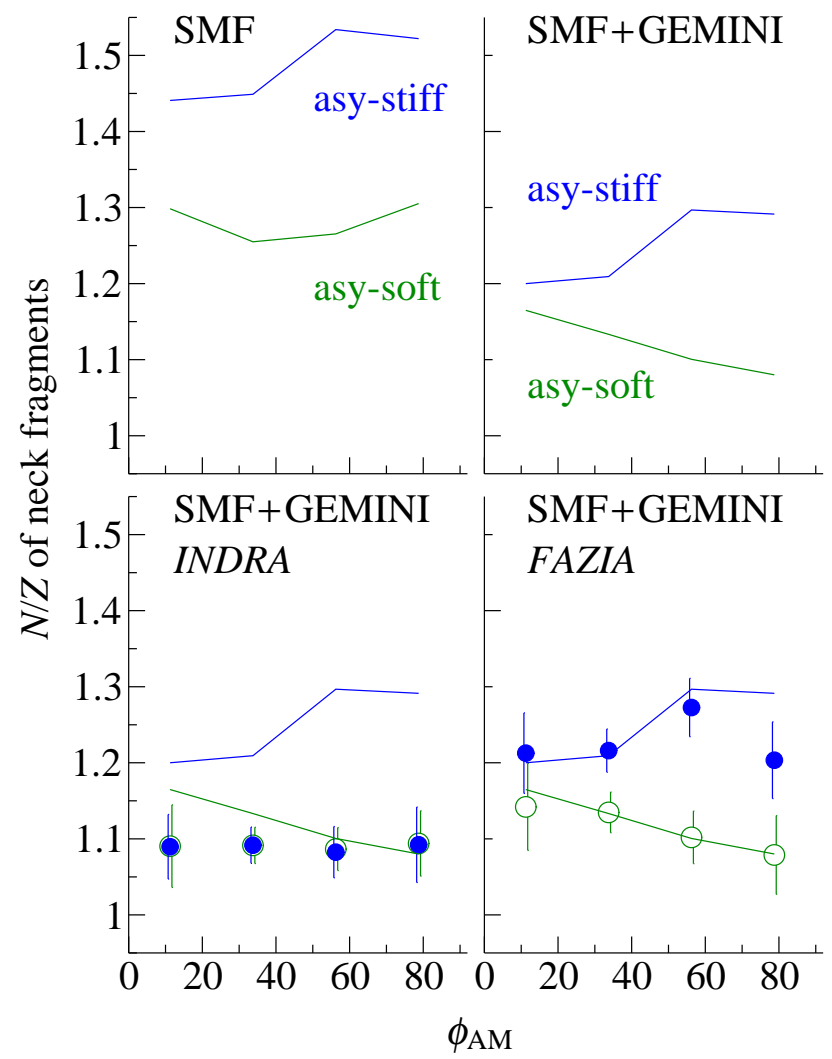

FIG. 11: (Color online) Isotopic composition of the neck fragment in ternary events, selected as defined in the text, as a function of $\phi_{\mathrm{AM}}$, for the system ${ }^{68} \mathrm{Ni}+{ }^{68} \mathrm{Ni}$ at $40 \mathrm{AMeV}$. The spectra are simulated for the hot system (SMF, upper left panel) and for the cold system (SMF+GEMINI, upper right panel). The spectra representing the cold system are simulated as for the two experimental approaches INDRA (lower left panel) and FAZIA (lower right panel). retical [39], as well as in experimental analyses [20]. It would be extremely interesting to pursue the investigation of the neck dynamics also at lower beam energy. Though the analysis is complicated by the reduced statistics of ternary events, fragments are expected to be less excited, opening the possibility to access more directly the genuine dynamical effects. Work is in progress in this direction.

\section{CONCLUSIONS}

In summary, we built a protocol for simulating reaction experiments which profit from next-generation radioactive-beam facilities and from innovative experimental solutions; these latter are specifically dedicated to the measurement of isotopic observables in exclusive experiments at low and intermediate energies. In this work, the systems ${ }^{68} \mathrm{Ni}+{ }^{68} \mathrm{Ni},{ }^{58} \mathrm{Ni}+{ }^{68} \mathrm{Ni}$ and ${ }^{58} \mathrm{Ni}+{ }^{58} \mathrm{Ni}$ at 15 and $40 \mathrm{MeV}$ per nucleon were simulated for a continuous distribution of impact parameters $b$ within the Stochastic Mean Field Model, with the addition of a secondary-decay process (described within the model GEMINI). The experimental conditions were simulated by supposing that the experimental observables are delivered either by the former-generation detector INDRA, or by a next-generation detector, which corresponds to the FAZIA project; such project, under development, is intended to build a $4 \pi$-array of silicon detectors which, beside measuring the nuclear charge of the fragments, are also sensitive to the mass and have low thresholds. Such simulation protocol was used to discuss new experimental solutions for probing the nuclear equation of state in heavy-ion collisions at Fermi energies.

Firstly, we studied the effect of the secondary decay. Such process, which creates a significant bias in the determination of the EOS properties, should be taken into account in the choice of the experimental strategy. According to our simulations, secondary-decay effects can be partially cured by studying exotic systems. However, even with exotic beams, secondary decay has to be controlled before a reliable extraction of the isovector equation of state can be obtained.

The strategy of constructing suitable combinations of isotopic observables (such as imbalance ratios), that was proposed for similarly prepared systems, appears to be an efficient way to reduce the effects of the secondary decay. Other possible solutions consist in selecting observables connected to fragments which are formed with insufficient excitation for feeding any relevant decay process, or uniquely connected to the initial phases of the reaction process, like in the preequilibrium stage.

Following this second approach, we identified two more probes which are negligibly affected by the secondary decay: the relative yields of preequilibrium high-energy light particles (we focused on neutrons and protons), and the isotopic composition of neck fragments in ternary events. Both observables would exhibit significantly dif- 
ferent signatures for the asy-stiff or the asy-soft behaviour of the symmetry energy, even when the secondary decay is accounted for. This is particularly evident in the case of the neutron-rich ${ }^{68} \mathrm{Ni}+{ }^{68} \mathrm{Ni}$ system, for which isospin effects are enhanced.

In this framework, observables related to the neck dynamics appear rather promising. Interesting effects have been revealed already with stable beams [20, 21], which may constitute one passage towards exotic-beam experiments. Moreover, it would be very interesting to extend this analysis to lower beam energies. We found that these signatures could be identified with an exclusive detector device which is compatible with the FAZIA project, and which is based on the simultaneous detection of charges and masses over a large solid angle. The principle of such an experimental approach has several advantages. First of all, the possibility of performing a complete event reconstruction through the data analysis allows to refine the criteria of centrality selection, which is a crucial point in the comparison between theoretical simulations and experimental data. Then, the measurement of the mass of all reaction products gives access to more sophisticated isospin-sensitive observables. Finally, we shall also stress that such experimental approach would be suited for analysing within the same experiment several probes for the symmetry energy, which are also sensitive to different stages of the reaction.

\section{ACKNOWLEDGEMENTS}

The efficiency diagram used to simulate the DEMON neutron telescopes was kindly provided by Frank Delaunay through a GEANT4 simulation.
[1] G.Poggi et al., FAZIA LoI for SPIRAL2, http://www.ganil.fr/research/developments/spiral2/

[2] C.Fuchs, Prog. Part. Nucl. Phys. 56 (2006) 1, and references therein.

[3] B.A. Li, L.W. Chen, C.M. Ko, Phys. Rep. 464, (2008) 113, and references therein.

[4] V. Baran, M. Colonna, V. Greco and M. Di Toro, Phys. Rep. 410 (2005) 335.

[5] D.V. Shetty, S.J. Yennello, and G.A. Souliotis, Phys. Rev. C 76, 024606 (2007).

[6] Z.Xiao, B.A.Li, L.W.Chen, G.C.Yong, M.Zhang, Phys.Rev.Lett. 102 (2009) 062502.

[7] M. Di Toro, V. Baran, M. Colonna, G. Ferini, T. Gaitanos, V. Giordano, V. Greco, Liu Bo, M. ZielinskaPfabe, S. Plumari, V. Prassa, C. Rizzo, J. Rizzo, H.H. Wolter, Prog. Part. Nucl. Phys. 62 (2009) 389.

[8] C.J.Horowitz and A.Schwenk, Nucl.Phys.A776 (2006) 55.

[9] G.Lehaut, F.Gulminelli, O.Lopez, Phys.Rev.Lett.102 (2009) 142503.

[10] M.B.Tsang, Yingxun Zhang, P.Danielewicz, M.Famiano, Zhuxia Li, W.G.Lynch, A.W.Steiner, Phys.Rev.Lett.102 (2009) 122701.

[11] T.X. Liu, M.J. van Goethem, X.D. Liu, W.G. Lynch, R. Shomin, W.P. Tan, M.B. Tsang, G. Verde, A. Wagner, H.F. Xi, H.S. Xu, M. Colonna, M. Di Toro, M. Zielinska-Pfabe, H. H. Wolter, L. Beaulieu, B. Davin, Y. Larochelle, T. Lefort, R. T. de Souza, R. Yanez, V. E. Viola, R.J. Charity, L.G. Sobotka, Phys.Rev. C69 (2004) 014603.

[12] D.V. Shetty, S.J. Yennello, A.S. Botvina, G.A. Souliotis, M. Jandel, E. Bell, A. Keksis, S. Soisson, B. Stein, J. Iglio, arXiv:nucl-ex/0603016

[13] W. D. Tian, Y. G. Ma, X. Z. Cai, D. Q. Fang, W. Guo, C. W. Ma, G. H. Liu, W. Q. Shen, Y. Shi, H. W. Wang, K. Wang, W. Xu, T. Z. Yan, Int.J.Mod.Phys.E17 (2008) 1705 .

[14] M. Colonna and M.B. Tsang, Eur. Phys. J. A30, 165 (2006).

[15] M. Di Toro, S.J. Yennello and B.-A. Li, Eur. Phys. J.
A30, 153 (2006).

[16] V.Baran, C.Rizzo, M.Colonna, M.Di Toro, D.Pierroutsakou, Phys.Rev.C79 (2009) 021603.

[17] M.B. Tsang, T.X. Liu, L. Shi, P. Danielewicz, C.K. Gelbke, X.D. Liu, W.G. Lynch, W.P. Tan, G. Verde, A. Wagner, and H.S. Xu, Phys. Rev. Lett. 92, 062701 (2004).

[18] E. Galichet, M. Colonna, B. Borderie, M. F. Rivet, Phys.Rev. C79 (2009)064615; Phys.Rev. C79 (2009)064614;

[19] L.-W. Chen, C.M. Ko and B.-A. Li, Phys. Rev. Lett. 94, 032701 (2005)

[20] E. De Filippo et al., Acta Physica Polonica B 40 (2009) 1199.

[21] F. Amorini, G. Cardella, G. Giuliani, M. Papa, C. Agodi, R. Alba, A. Anzalone, I. Berceanu, S. Cavallaro, M. B. Chatterjee, R. Coniglione, E. De Filippo, A. Di Pietro,1 E. Geraci, L. Grassi, A. Grzeszczuk, P. Figuera, E. La Guidara, G. Lanzalone, N. Le Neindre, I. Lombardo, C. Maiolino, A. Pagano, S. Pirrone, G. Politi, A. Pop, F. Porto, F. Rizzo, P. Russotto, D. Santonocito, P. Sapienza, and G. Verde, Phys. Rev. Lett. 102, 112701 (2009).

[22] 'Mid-term Status Report of WP5-Task 5.3 (FAZIA) for SPIRAL2 PP (2009)', http://fazia.in2p3.fr

[23] J. Pouthas, B. Borderie, E. Plagnol, M.F. Rivet, F. SaintLaurent, J.C. Steckmeyer, G. Auger, Ch.O. Bacri, S. Barbey, A. Barbier, A. Benkirane, J. Benlliure, P. Bourgault, P. Box, R. Bzyl, D. Charlet, J.L. Charvet, A. Chbihi, T. Clerc, N. Copinet, D. Cussol, M. Engrand, J.M. Gautier, Y. Huguet, O. Jouniaux, J.L. Laville, P. Lelong, P. Osrin, L. Olivier, S. Pierre, B. Piquet, E. Plaige, B. Raine, A. Richard, J. Ropert, C. Spitaels, L. Stab, D. Sznajderman, L. Tassan-Got, J. Tillier, M. Tripon, P. Vallerand, P. Volkov, J.P. Wieleczko and G. Wittwer, Nucl. Inst. Meth. A 357, 418 (1995).

[24] J. Pouthas, A. Bertaut, B. Borderie, P. Bourgault, B. Cahan, G. Carles, D. Charlet, D. Cussol, R. Dayras, M. Engrand, O. Jouniaux, P. Le Botlan, A. Leconte, P. Lelong, L. Martina, P. Mosrin, L. Olivier, J.P. Passerieux, B. Piquet, E. Plagnol, E. Plaige, B. Raine, A. Richard, 
F. Saint-Laurent, C. Spitaels, J. Tillier, M. Tripon, P. Vallerand, P. Volkov and G. Wittwer, Nucl. Inst. Meth. A 369, 222 (1996).

[25] A. Pagano et al., Nucl. Phys. A734 (2004) 504.

[26] J.Rizzo, Ph.Chomaz, M.Colonna, Nucl. Phys. A806 (2008) 40.

[27] J. Rizzo, M. Colonna, V. Baran, M. Di Toro, H.H. Wolter, M. Zielinska-Pfabe, Nucl. Phys. A806 (2008) 79.

[28] J. Bartel, P. Quentin, M. Brack, C. Guet and H.-B. Håkansson, Nucl. Phys. A386 (1982) 79.

[29] R.J. Charity, M.A. McMahan, G.J. Wozniak, R.J. McDonald, L.G. Moretto, D.G. Sarantites, L.G. Sobotka, G. Guarino, A. Pantaleo, L. Fiore, A. Gobbi and K.D. Hildenbrand, Nucl. Phys. A483, 371 (1988).

[30] A. Ono and J. Randrup, Eur. Phys. J. A30, 109 (2006).

[31] P.Napolitani and the FAZIA simulation group, 'Panforte package', http://fazia.in2p3.fr

[32] I. Tilquin, Y. El Masri, M. Parlog, P. Collon, M. Hadri, T. Keutgen, J. Lehmann, P. Leleux, P. Lipnik, A. Ninane,
F. Hanappe, G. Bizard, D. Durand, P. Mosrin, J. Peter, R. Regimbart and B Tamain, Nucl. Instr. and Methods A 365, 446 (1995).

[33] A. Stolz et al., Nucl. Phys. A 746 (2004) 54c.

[34] J. Benlliure, M. Fernandez-Ordonez, L. Audouin, A. Boudard, E. Casarejos, J. E. Ducret, T. Enqvist, A. Heinz, D. Henzlova, V. Henzl, A. Kelic, S. Leray, P. Napolitani, J. Pereira, F. Rejmund, M. V. Ricciardi, K. -H. Schmidt, C. Schmitt, C. Stephan, L. Tassan-Got, C. Volant, C. Villagrasa, and O. Yordanov Phys. Rev. C 78 (2008) 054605.

[35] B.A. Li, L.W. Chen, G.C. Yong, W. Zuo, Phys. Lett. B 634 (2006) 378.

[36] Y. Zhang, P. Danielewicz, M. Famiano, Z. Li, W.G. Lynch, M.B. Tsang, Phys. Lett. B 664 (2008) 145.

[37] E. De Filippo et al., Phys. Rev. C 71 (2005) 064604.

[38] F. Rami et al., Phys. Rev. Lett. 84, 1120 (2000).

[39] R. Lionti et al., Phys. Lett.B625, 33 (2005). 